Mathematical Research Letters 11, 187-196 (2004)

\title{
ARITHMETIC PROPERTIES OF PERIODIC MAPS
}

\author{
ZHI-WeI Sun
}

\begin{abstract}
Let $\psi_{1}, \ldots, \psi_{k}$ be periodic maps from $\mathbb{Z}$ to a field of characteristic $p$ (where $p$ is zero or a prime). Assume that positive integers $n_{1}, \ldots, n_{k}$ not divisible by $p$ are their periods respectively. We show that $\psi_{1}+\cdots+\psi_{k}$ is constant if $\psi_{1}(x)+\cdots+\psi_{k}(x)$ equals a constant for $|S|$ consecutive integers $x$ where $S=\bigcup_{s=1}^{k}\left\{r / n_{s}: r=0, \ldots, n_{s}-1\right\}$. We also present some new results on finite systems of arithmetic sequences.
\end{abstract}

\section{Introduction}

For $a \in \mathbb{Z}$ and $n \in \mathbb{Z}^{+}=\{1,2,3, \ldots\}$ we call

$$
a(n)=a+n \mathbb{Z}=\{a+n x: x \in \mathbb{Z}\}
$$

an arithmetic sequence with modulus $n$. For a finite system

$$
A=\left\{a_{s}\left(n_{s}\right)\right\}_{s=1}^{k}
$$

of such sequences, the covering function $w_{A}: \mathbb{Z} \rightarrow \mathbb{Z}$ given by

$$
w_{A}(x)=\left|\left\{1 \leqslant s \leqslant k: x \in a_{s}\left(n_{s}\right)\right\}\right|
$$

is obviously periodic modulo the least common multiple $\left[n_{1}, \ldots, n_{k}\right]$ of all the moduli $n_{1}, \ldots, n_{k}$. If $w_{A}(x) \leqslant 1$ for all $x \in \mathbb{Z}$ (i.e., $a_{i}\left(n_{i}\right) \cap a_{j}\left(n_{j}\right)=\emptyset$ if $1 \leqslant i<j \leqslant k$ ), then we say that (1.1) is disjoint. When $w_{A}(x) \geqslant 1$ for all $x \in \mathbb{Z}$ (i.e., $\left.\bigcup_{s=1}^{k} a_{s}\left(n_{s}\right)=\mathbb{Z}\right),(1.1)$ is called a cover of $\mathbb{Z}$.

A famous result of H. Davenport, L. Mirsky, D. Newman and R. Radó (cf. [NZ]) states that if (1.1) is a disjoint cover of $\mathbb{Z}$ with $1<n_{1} \leqslant \cdots \leqslant n_{k-1} \leqslant n_{k}$ then we must have $n_{k-1}=n_{k}$. In $1958 \mathrm{~S}$. K. Stein [St] conjectured that if (1.1) is disjoint with $1<n_{1}<\cdots<n_{k}$ then there exists an integer $x \notin \bigcup_{s=1}^{k} a_{s}\left(n_{s}\right)$ with $1 \leqslant x \leqslant 2^{k}$. In 1965 P. Erdös [E2] offered a prize for a proof of his

Received November 17, 2002, Revised March 2, 2004.

2000 Mathematics Subject Classification. Primary 11B25; Secondary 11A07, 11A25, 11Y16, $68 \mathrm{Q} 25$.

Supported by the Teaching and Research Award Fund for Outstanding Young Teachers in Higher Education Institutions of MOE, and the Key Program of the National Natural Science Foundation of P. R. China. 
following stronger conjecture (see [E1]): (1.1) forms a cover of $\mathbb{Z}$ if it covers those integers from 1 to $2^{k}$. (The above $2^{k}$ is best possible because $\left\{2^{s-1}\left(2^{s}\right)\right\}_{s=1}^{k}$ covers $1, \ldots, 2^{k}-1$ but does not cover any multiple of $2^{k}$.) In 1969-1970 R. B. Crittenden and C. L. Vanden Eynden [CV1, CV2] supplied a long and awkward proof of the Erdős conjecture for $k \geqslant 20$.

Let $m$ be a positive integer. In [Su4, Su5] the author called (1.1) an $m$-cover of $\mathbb{Z}$ if $w_{A}(x) \geqslant m$ for all $x \in \mathbb{Z}$, and an exact $m$-cover of $\mathbb{Z}$ if $w_{A}(x)=m$ for all $x \in \mathbb{Z}$. Recently the author [Su10] found that $m$-covers of $\mathbb{Z}$ are closely related to subset sums in a field and zero-sum problems on abelian groups.

Here is a result of [Su4, Su5] stronger than Erdős' conjecture: (1.1) forms an $m$-cover of $\mathbb{Z}$ if it covers $\left|\left\{\left\{\sum_{s \in I} m_{s} / n_{s}\right\}: I \subseteq\{1, \ldots, k\}\right\}\right|$ consecutive integers at least $m$ times, where the given $m_{1}, \ldots, m_{k} \in \mathbb{Z}^{+}$are relatively prime to $n_{1}, \ldots, n_{k}$ respectively. (As usual the fractional part of a real number $x$ is denoted by $\{x\}$.) In $[\mathrm{Su} 5]$ the author asked whether we have a similar result for exact $m$-covers of $\mathbb{Z}$. The answer is actually negative, moreover there is no constant $c(k, m) \in \mathbb{Z}^{+}$such that (1.1) forms an exact $m$-cover of $\mathbb{Z}$ whenever it covers $c(k, m)$ consecutive integers exactly $m$ times. In fact, if (1.1) is an exact $m$-cover of $\mathbb{Z}$, then for any integer $N>1$ the system $\left\{a_{1}\left(n_{1}\right), \ldots, a_{k}\left(n_{k}\right), 0(N)\right\}$ covers $1, \ldots, N-1$ exactly $m$ times but it covers 0 exactly $m+1$ times! (This observation is due to the author's student H. Pan.)

For an assertion $P$ we adopt Iverson's notation

$$
\llbracket P \rrbracket= \begin{cases}1 & \text { if } P \text { holds } \\ 0 & \text { otherwise }\end{cases}
$$

Observe that $w_{A}(x)=\sum_{s=1}^{k} \psi_{s}(x)$ where $\psi_{s}(x)=\llbracket n_{s} \mid x-a_{s} \rrbracket$ is periodic modulo $n_{s}$.

Our first result is completely new!

Theorem 1.1. Let $F$ be a field of characteristic $p$ where $p$ is zero or a prime. Let $n_{1}, \ldots, n_{k}$ be positive integers not divisible by $p$, and let $\psi_{1}, \ldots, \psi_{k}$ be maps from $\mathbb{Z}$ to $F$ with periods $n_{1}, \ldots, n_{k}$ respectively. Then $\psi_{1}+\cdots+\psi_{k}=0$ if $\psi_{1}(x)+\cdots+\psi_{k}(x)=0$ for $\sum_{d \in D} \varphi(d)$ consecutive integers $x$, where $\varphi$ is Euler's totient function, $D=\bigcup_{s=1}^{k} D\left(n_{s}\right)$, and $D(n)$ denotes the set of positive divisors of $n \in \mathbb{Z}^{+}$.

Remark 1.1. Clearly $\sum_{d \in D} \varphi(d)$ in Theorem 1.1 equals the cardinality of the set

$$
\bigcup_{d \in D}\left\{\frac{c}{d}: 0 \leqslant c<d \text { and }(c, d)=1\right\}=\bigcup_{s=1}^{k}\left\{\frac{r}{n_{s}}: r=0,1, \ldots, n_{s}-1\right\} \text {, }
$$

where $(c, d)$ is the greatest common divisor of $c$ and $d$. The result stated in the abstract is equivalent to Theorem 1.1 since a constant can be viewed as a function on $\mathbb{Z}$ periodic $\bmod 1$. 
Corollary 1.1. Let $w(x)$ be a function from $\mathbb{Z}$ to $\mathbb{Z}$ with period $n_{0} \in \mathbb{Z}^{+}$. Then $w(x)$ is the covering function of (1.1) if $w_{A}(x)=w(x)$ for

$$
\left|\bigcup_{s=0}^{k}\left\{0, \frac{1}{n_{s}}, \ldots, \frac{n_{s}-1}{n_{s}}\right\}\right| \leqslant n_{0}+n_{1}+\cdots+n_{k}-k
$$

consecutive integers $x$. In particular, (1.1) forms an exact $m$-cover of $\mathbb{Z}$ if it covers $\left|\bigcup_{s=1}^{k}\left\{r / n_{s}: r=0, \ldots, n_{s}-1\right\}\right|$ consecutive integers exactly $m$ times.

Proof. Let $D=\bigcup_{s=0}^{k} D\left(n_{s}\right)$. As

$$
\psi(x):=w_{A}(x)-w(x)=-w(x)+\sum_{s=1}^{k} \llbracket n_{s} \mid x-a_{s} \rrbracket
$$

vanishes for $\left|\bigcup_{s=0}^{k}\left\{r / n_{s}: r=0, \ldots, n_{s}-1\right\}\right|=\sum_{d \in D} \varphi(d)$ consecutive integers $x$, we have $\psi(x)=0$ for all $x \in \mathbb{Z}$ by Theorem 1.1. When $n_{0}=1$ and $w(x)=$ $m \in \mathbb{Z}^{+}$, this yields the latter result in Corollary 1.1.

Remark 1.2. The problem whether a given $A=\left\{a_{s}\left(n_{s}\right)\right\}_{s=1}^{k}$ forms a cover of $\mathbb{Z}$ is known to be co-NP-complete. (See, e.g. [GJ] and [T].) However, Corollary 1.1 indicates that we can check whether system $A$ has a given covering function in polynomial time! In 1997 the author [Su6] showed that if (1.1) covers all the integers the same number of times then

$$
\left\{\sum_{s \in I} \frac{1}{n_{s}}: I \subseteq\{1, \ldots, k\}\right\} \supseteq \bigcup_{s=1}^{k}\left\{\frac{r}{n_{s}}: r=0, \ldots, n_{s}-1\right\} .
$$

Example 1.1. Let (1.1) be an exact $m$-cover of $\mathbb{Z}$, and let $n$ be an integer greater than $n_{k}$. Then the system

$$
A^{\prime}=\left\{a_{1}\left(n_{1}\right), \ldots, a_{k-1}\left(n_{k-1}\right), a_{k}+n_{k}(n)\right\}
$$

covers each of the consecutive integers $a_{k}+1, \ldots, a_{k}+2 n_{k}-1$ exactly $m$ times but it does not cover $a_{k}$ or $a_{k}+2 n_{k}$ exactly $m$ times. For example, $B=$ $\{1(2), 2(4), 0(4)\}$ is a disjoint cover of $\mathbb{Z}$, thus $B^{\prime}=\{1(2), 2(4), 4(6)\}$ covers $1, \ldots, 7$ exactly once but it is not a disjoint cover of $\mathbb{Z}$. Note that the set $\bigcup_{n \in\{2,4,6\}}\{r / n: r=0, \ldots, n-1\}$ just has 8 elements.

Corollary 1.2. Let (1.1) be a system of arithmetic sequences, and let $m$ be any integer greater than $k-f\left(\left[n_{1}, \ldots, n_{k}\right]\right)$. (The function $f$ is given by $f(1)=0$ and $f\left(\prod_{i=1}^{r} p_{i}\right)=\sum_{i=1}^{r}\left(p_{i}-1\right)$ where $p_{1}, \ldots, p_{r}$ are primes.) Then there is an $x \in\{0,1, \ldots,|S|-1\}$ such that $w_{A}(x) \neq m$ where $S=\bigcup_{s=1}^{k}\left\{r / n_{s}: r=\right.$ $\left.0,1, \ldots, n_{s}-1\right\}$.

Proof. If (1.1) is an exact $m$-cover of $\mathbb{Z}$, then $k \geqslant m+f\left(\left[n_{1}, \ldots, n_{k}\right]\right)$ by Corollary 4.5 of [Su7]. Thus, in view of the condition, (1.1) does not form an exact $m$-cover of $\mathbb{Z}$ and hence the desired result follows from Corollary 1.1.

Our next theorem extends some earlier work in [Su4, Su5]. 
Theorem 1.2. Let $n_{1}, \ldots, n_{k}$ be positive integers, and let $R_{1}, \ldots, R_{k}$ be finite subsets of $\mathbb{Z}$. For $s=1, \ldots, k$, let $c_{\text {st }}$ lie in the complex field $\mathbb{C}$ for each $t \in R_{s}$, and set

$$
X_{s}=\left\{x \in \mathbb{Z}: \sum_{t \in R_{s}} c_{s t} e^{2 \pi i \frac{t}{n_{s}} x}=0\right\}
$$

If the system $\left\{X_{s}\right\}_{s=1}^{k}$ covers $W$ consecutive integers at least $m$ times where $1 \leqslant m \leqslant k$ and

$$
W=\max _{\substack{I \subseteq\{1, \ldots, k\} \\|I|=k-m+1}}\left|\left\{\left\{\sum_{s \in I} \frac{r_{s}}{n_{s}}\right\}: r_{s} \in R_{s}\right\}\right| \leqslant \max _{\substack{I \subseteq\{1, \ldots, k\} \\|I|=k-m+1}} \prod_{s \in I}\left|R_{s}\right|,
$$

then it covers every integer at least $m$ times.

Corollary 1.3. Let (1.1) be a system of arithmetic sequences, and let $m_{1}, \ldots, m_{k}$ be integers relatively prime to $n_{1}, \ldots, n_{k}$ respectively. Let $l$ be any nonnegative integer with $w_{A}(x) \geqslant l$ for all $x \in \mathbb{Z}$, and set

$$
W_{l}=\max _{\substack{I \subseteq\{1, \ldots, k\} \\|I|=k-l}}\left|\left\{\left\{\sum_{s \in J} \frac{m_{s}}{n_{s}}\right\}: J \subseteq I\right\}\right| \leqslant 2^{k-l} .
$$

Then the covering function $w_{A}(x)$ takes its minimum on every set of $W_{l}$ consecutive integers.

Proof. Without loss of generality we may assume that $1 \leqslant m_{s} \leqslant n_{s}$ for all $s=1, \ldots, k$. As $m(A)=\min _{x \in \mathbb{Z}} w_{A}(x) \geqslant l$ and $W_{l} \geqslant W_{m(A)}$, it suffices to work with $l=m(A)$ below.

The case $l=k$ is trivial, so we let $l<k$. Set $c_{s 0}=1$ and $c_{s m_{s}}=-e^{-2 \pi i a_{s} m_{s} / n_{s}}$ for $s=1, \ldots, k$. Since $m_{s}$ and $n_{s}$ are relatively prime,

$$
X_{s}:=\left\{x \in \mathbb{Z}: c_{s 0} e^{2 \pi i \frac{0}{n_{s}} x}+c_{s m_{s}} e^{2 \pi i \frac{m_{s}}{n_{s}} x}=0\right\}=a_{s}\left(n_{s}\right) .
$$

Applying Theorem 1.2 with $m=l+1$ and $R_{s}=\left\{0, m_{s}\right\}(1 \leqslant s \leqslant k)$, we immediately get the desired result.

Remark 1.3. (a) [Su9] contains some other interesting results on the covering function of (1.1). (b) $W_{l}$ in (1.6) might be smaller than its value in the case $m_{1}=\cdots=m_{k}=1$. Let $n_{1}=3, n_{2}=5$ and $n_{3}=15$. Set

$$
W_{0}\left(m_{1}, m_{2}, m_{3}\right)=\left|\left\{\left\{\sum_{s \in J} \frac{m_{s}}{n_{s}}\right\}: J \subseteq\{1,2,3\}\right\}\right|
$$

for $m_{1}, m_{2}, m_{3} \in \mathbb{Z}$. Then $W_{0}(1,1,2)=7<W_{0}(1,1,1)=8$.

Our third theorem characterizes the least period of a covering function. 
Theorem 1.3. Let $\lambda_{s} \in \mathbb{C}, a_{s} \in \mathbb{Z}$ and $n_{s} \in \mathbb{Z}^{+}$for $s=1, \ldots, k$. Then the smallest positive period $n_{0}$ of the (weighted) covering function

$$
w(x)=\sum_{s=1}^{k} \lambda_{s} \llbracket n_{s} \mid x-a_{s} \rrbracket
$$

is the least $n \in \mathbb{Z}^{+}$such that $\alpha n \in \mathbb{Z}$ for all those $\alpha \in[0,1)$ with

$$
\sum_{\substack{1 \leqslant s \leqslant k \\ \alpha n_{s} \in \mathbb{Z}}} \frac{\lambda_{s}}{n_{s}} e^{2 \pi i \alpha a_{s}} \neq 0 .
$$

Remark 1.4. Under the condition of Theorem 1.3, it can be easily checked that $\sum_{x=0}^{N-1} w(x) / N=\sum_{s=1}^{k} \lambda_{s} / n_{s}$ where $N=\left[n_{1}, \ldots, n_{k}\right]$. If $w(x)=0$ for all $x \in \mathbb{Z}$, then $n_{0}=1$ and hence

$$
\sum_{\substack{s=1 \\ \alpha n_{s} \in \mathbb{Z}}}^{k} \frac{\lambda_{s}}{n_{s}} e^{2 \pi i \alpha a_{s}}=0 \quad \text { for all } \alpha \in[0,1) .
$$

This was first obtained by the author [Su2] in 1991 via an analytic method, and the converse was proved in [Su3]. In [Su8] the author determined those functions $f: \bigcup_{n \in \mathbb{Z}^{+}} \mathbb{Z} / n \mathbb{Z} \rightarrow \mathbb{C}$ such that $\sum_{s=1}^{k} \lambda_{s} f\left(a_{s}+n_{s} \mathbb{Z}\right)$ only depends on the covering function $w(x)$, this was announced by the author [Su1] in 1989.

Let $l$ be a positive integer, and let

$$
\mathbb{Z}^{l}=\left\{\vec{x}=\left\langle x_{1}, \ldots, x_{l}\right\rangle: x_{1}, \ldots, x_{l} \in \mathbb{Z}\right\}
$$

be the direct sum of $l$ copies of the $\operatorname{ring} \mathbb{Z}$. For $\vec{x}, \vec{y} \in \mathbb{Z}^{l}$, we use $\vec{x} \mid \vec{y}$ to mean that $\vec{y}=\vec{q} \vec{x}=\left\langle q_{1} x_{1}, \ldots, q_{l} x_{l}\right\rangle$ for some $\vec{q} \in \mathbb{Z}^{l}$. A function $\Psi: \mathbb{Z}^{l} \rightarrow \mathbb{C}$ is said to be periodic modulo $\vec{n} \in \mathbb{Z}^{l}$ if $\Psi(\vec{x})=\Psi(\vec{y})$ whenever $\vec{x}-\vec{y}=\left\langle x_{1}-y_{1}, \ldots, x_{l}-y_{l}\right\rangle$ is divisible by $\vec{n}$. For $x_{1}, \ldots, x_{l} \in \mathbb{Z}$, we also use $\left[x_{t}\right]_{1 \leqslant t \leqslant l}$ to denote the least common multiple of $x_{1}, \ldots, x_{l}$.

Theorem 1.4. Let $\lambda_{s} \in \mathbb{C}, \vec{a}_{s} \in \mathbb{Z}^{l}$ and $\vec{n}_{s} \in\left(\mathbb{Z}^{+}\right)^{l}$ for $s=1, \ldots, k$ where $l \in \mathbb{Z}^{+}$. Suppose that the function

$$
w(\vec{x})=\sum_{s=1}^{k} \lambda_{s} \llbracket \vec{n}_{s} \mid \vec{x}-\vec{a}_{s} \rrbracket
$$

is periodic modulo $\vec{n}_{0} \in\left(\mathbb{Z}^{+}\right)^{l}$. Let $\vec{d} \in\left(\mathbb{Z}^{+}\right)^{l}, \vec{d} \nmid \vec{n}_{0}$ and

$$
I(\vec{d})=\left\{1 \leqslant s \leqslant k: \vec{d} \mid \vec{n}_{s}\right\} \neq \emptyset .
$$




$$
\begin{aligned}
& \text { If } \sum_{s \in I(\vec{d})} \lambda_{s} /\left(n_{s 1} \cdots n_{s l}\right) \neq 0, \text { then } \\
& \qquad|I(\vec{d})| \geqslant\left|\left\{\left\{\sum_{t=1}^{l} \frac{a_{s t}}{d_{t}}\right\}: s \in I(\vec{d})\right\}\right| \geqslant \min _{\substack{0 \leqslant s \leqslant k \\
\vec{d} \nmid \vec{n}_{s}}}\left[\frac{d_{t}}{\left(d_{t}, n_{s t}\right)}\right]_{1 \leqslant t \leqslant l} \geqslant p\left(d_{1} \cdots d_{l}\right)
\end{aligned}
$$

where we use $p(m)$ to denote the least prime divisor of an integer $m>1$.

Remark 1.5. Theorem 1.4 is a generalization of the main result of [Su2] which corresponds to the case $l=1$ and improves the Znám-Newman result [N].

Corollary 1.4. Let $\lambda_{s} \in \mathbb{C} \backslash\{0\}, \vec{a}_{s} \in \mathbb{Z}^{l}$ and $\vec{n}_{s} \in\left(\mathbb{Z}^{+}\right)^{l}$ for $s=1, \ldots, k$ where $l \in \mathbb{Z}^{+}$. Suppose that all those moduli $\vec{n}_{s}$ which are maximal with respect to divisibility are distinct. Then the function $w(\vec{x})$ given by $(1.8)$ is periodic modulo $\vec{n}_{0} \in\left(\mathbb{Z}^{+}\right)^{l}$ if and only if $\vec{n}_{0}$ is divisible by all the moduli $\vec{n}_{1}, \ldots, \vec{n}_{k}$.

Proof. If $\vec{n}_{s} \mid \vec{n}_{0}$ for all $s=1, \ldots, k$, then the function $w(\vec{x})$ is obviously periodic $\bmod \vec{n}_{0}$.

Now suppose that $w(\vec{x})$ is periodic modulo $\vec{n}_{0}$ but not all the moduli divide $\vec{n}_{0}$. Then there exists a maximal modulus $\vec{n}_{r}$ with respect to divisibility such that $\vec{n}_{r} \nmid \vec{n}_{0}$. By the condition,

$$
I\left(\vec{n}_{r}\right):=\left\{1 \leqslant s \leqslant k: \vec{n}_{r} \mid \vec{n}_{s}\right\}=\left\{1 \leqslant s \leqslant k: \vec{n}_{s}=\vec{n}_{r}\right\}=\{r\} .
$$

On the other hand, by Theorem 1.4 we should have $\left|I\left(\vec{n}_{r}\right)\right| \geqslant p\left(n_{r 1} \cdots n_{r l}\right)$. The contradiction ends our proof.

Remark 1.6. Corollary 1.4 in the case $l=1$ was essentially established by $\check{\mathrm{S}}$. Porubský $[\mathrm{P}]$.

\section{Proofs of Theorems 1.1-1.4}

Lemma 2.1. Let $c_{1}, \ldots, c_{n}$ lie in a field $F$, and let $z_{1}, \ldots, z_{n}$ be distinct elements of $F \backslash\{0\}$. If $\sum_{j=1}^{n} c_{j} z_{j}^{x}$ vanishes for $n$ consecutive integers $x$, then it vanishes for all $x \in \mathbb{Z}$.

Proof. Suppose that $\sum_{j=1}^{n} c_{j} z_{j}^{h+i-1}=0$ for every $i=1, \ldots, n$ where $h \in \mathbb{Z}$. Since the Vandermonde determinant

$$
\left\|z_{j}^{i-1}\right\|_{1 \leqslant i, j \leqslant n}=\left|\begin{array}{cccc}
1 & 1 & \ldots & 1 \\
z_{1} & z_{2} & \ldots & z_{n} \\
\vdots & \vdots & \ddots & \vdots \\
z_{1}^{n-1} & z_{2}^{n-1} & \ldots & z_{n}^{n-1}
\end{array}\right|=\prod_{1 \leqslant i<j \leqslant n}\left(z_{j}-z_{i}\right)
$$

does not vanish, by Cramer's rule we have $c_{j} z_{j}^{h}=0$ and hence $c_{j}=0$ for all $j=1, \ldots, n$. Therefore $\sum_{j=1}^{n} c_{j} z_{j}^{x}=0$ for any $x \in \mathbb{Z}$. 
Proof of Theorem 1.1. As $p$ does not divide $N=\left[n_{1}, \ldots, n_{k}\right]$, the equation $x^{N}-1=0$ has $N$ distinct roots in the algebraic closure $E$ of the field $F$. The multiplicative group $\left\{\zeta \in E: \zeta^{N}=1\right\}$ of order $N$ is cyclic, so $E$ contains an element $\zeta$ of multiplicative order $N$. For $a \in \mathbb{Z}$ and $1 \leqslant s \leqslant k$, we have the geometric series

$$
\frac{1}{n_{s}} \sum_{r=0}^{n_{s}-1} \zeta^{\frac{N}{n_{s}} a r}=\llbracket n_{s} \mid a \rrbracket
$$

Therefore

$$
\begin{aligned}
\sum_{s=1}^{k} \psi_{s}(x) & =\sum_{s=1}^{k} \sum_{a=0}^{n_{s}-1} \llbracket n_{s} \mid a-x \rrbracket \psi_{s}(a) \\
& =\sum_{s=1}^{k} \sum_{a=0}^{n_{s}-1} \frac{1}{n_{s}} \sum_{r=0}^{n_{s}-1} \zeta^{\frac{N}{n_{s}}(a-x) r} \psi_{s}(a) \\
& =\sum_{s=1}^{k} \frac{1}{n_{s}} \sum_{a=0}^{n_{s}-1} \psi_{s}(a) \sum_{\substack{0 \leqslant \alpha<1 \\
\alpha n_{s} \in \mathbb{Z}}} \zeta^{\alpha N(a-x)} \\
& =\sum_{\alpha \in S}\left(\zeta^{-\alpha N}\right)^{x}\left(\sum_{s=1}^{k} \frac{\llbracket \alpha n_{s} \in \mathbb{Z} \rrbracket}{n_{s}} \sum_{a=0}^{n_{s}-1} \psi_{s}(a) \zeta^{\alpha N a}\right)
\end{aligned}
$$

where $S$ is the set

$$
\left\{\alpha \in[0,1): \alpha n_{s} \in \mathbb{Z} \text { for some } 1 \leqslant s \leqslant k\right\}=\bigcup_{s=1}^{k}\left\{\frac{r}{n_{s}}: r=0, \ldots, n_{s}-1\right\} .
$$

As those $\zeta^{-\alpha N}$ with $\alpha \in S$ are distinct, applying Lemma 2.1 we find that $\sum_{s=1}^{k} \psi_{s}(x)=0$ for $|S|$ consecutive integers $x$ if and only if $\sum_{s=1}^{k} \psi_{s}(x)=0$ for all $x \in \mathbb{Z}$. By Remark 1.1, $|S|=\sum_{d \in D} \varphi(d)$. This concludes the proof.

Proof of Theorem 1.2. Clearly an integer $x$ is covered by $\left\{X_{s}\right\}_{s=1}^{k}$ at least $m$ times if and only if $x$ is covered by $\left\{X_{s}\right\}_{s \in I}$ for all $I \subseteq\{1, \ldots, k\}$ with $|I|=k-m+1$.

Now let $I \subseteq\{1, \ldots, k\}$ and $|I|=k-m+1$. For any $x \in \mathbb{Z}$, we have

$$
\begin{aligned}
\prod_{s \in I} \sum_{t \in R_{s}} c_{s t} e^{2 \pi i \frac{t}{n_{s}} x} & =\sum_{r_{s} \in R_{s} \text { for }}\left(\prod_{s \in I} c_{s r_{s}}\right) e^{2 \pi i x \sum_{s \in I} r_{s} / n_{s}} \\
& =\sum_{\theta \in R(I)} C_{I, \theta} e^{2 \pi i \theta x}
\end{aligned}
$$

where

$$
R(I)=\left\{\left\{\sum_{s \in I} \frac{r_{s}}{n_{s}}\right\}: r_{s} \in R_{s}\right\} \text { and } C_{I, \theta}=\sum_{\substack{r_{s} \in R_{s} \text { for } \\\left\{\sum_{s \in I} r_{s} / n_{s}\right\}=\theta}} \prod_{s \in I} c_{s r_{s}} .
$$


Since those $e^{2 \pi i \theta}$ with $\theta \in R(I)$ are distinct, by Lemma 2.1 the system $\left\{X_{s}\right\}_{s \in I}$ covers $|R(I)|$ consecutive integers $x$ if and only if it covers all $x \in \mathbb{Z}$.

In view of the above, we immediately obtain the desired result.

Proof of Theorem 1.3. Let $S=\left\{0 \leqslant \alpha<1: \alpha n_{s} \in \mathbb{Z}\right.$ for some $\left.1 \leqslant s \leqslant k\right\}$ and

$$
T=\left\{0 \leqslant \alpha<1: c_{\alpha}=\sum_{\substack{1 \leqslant s \leqslant k \\ \alpha n_{s} \in \mathbb{Z}}} \frac{\lambda_{s}}{n_{s}} e^{2 \pi i \alpha a_{s}} \neq 0\right\}
$$

For each $s=1, \ldots, k$ the arithmetical function $\psi_{s}(x)=\lambda_{s} \llbracket n_{s} \mid x-a_{s} \rrbracket$ is periodic modulo $n_{s}$. By the proof of Theorem 1.1, for any $x \in \mathbb{Z}$ we have

$$
w(x)=\sum_{s=1}^{k} \lambda_{s} \llbracket n_{s} \mid x-a_{s} \rrbracket=\sum_{\alpha \in S} e^{-2 \pi i \alpha x} c_{\alpha}=\sum_{\alpha \in T} e^{-2 \pi i \alpha x} c_{\alpha} .
$$

Let $n$ be the least positive integer such that $\alpha n \in \mathbb{Z}$ for all $\alpha \in T$. By the above, $w(x)=w(x+n)$ for all $x \in \mathbb{Z}$. Thus $n_{0} \mid n$.

If $T=\emptyset$, then $n=1$ and hence $n_{0}=n$. In the case $T \neq \emptyset$, we have

$$
0=w(x)-w\left(x+n_{0}\right)=\sum_{\alpha \in T} e^{-2 \pi i \alpha x}\left(1-e^{-2 \pi i \alpha n_{0}}\right) c_{\alpha}
$$

for every $x=0, \ldots,|T|-1$, and hence $\left(1-e^{-2 \pi i \alpha n_{0}}\right) c_{\alpha}=0$ for any $\alpha \in T$ (Vandermonde). Now that $\alpha n_{0} \in \mathbb{Z}$ (i.e., $e^{-2 \pi i \alpha n_{0}}=1$ ) for all $\alpha \in T$, we have $n_{0} \geqslant n$ and thus $n_{0}=n$.

The proof of Theorem 1.3 is now complete.

Proof of Theorem 1.4. Let $\vec{c}$ be any vector in $\mathbb{Z}^{l}$ with $\vec{d} \nmid \vec{c}_{0}$. Then, for some $1 \leqslant$ $r \leqslant l$ we have $d_{r} \nmid c_{r} n_{0 r}$. Note that $\vec{n}_{0}$ divides the vector $\left\langle 0, \ldots, 0, n_{0 r}, 0, \ldots, 0\right\rangle$. For any $x_{1}, \ldots, x_{r-1}, x_{r+1}, \ldots, x_{l} \in \mathbb{Z}$, since

$$
\sum_{s=1}^{k}\left(\lambda_{s} \prod_{\substack{t=1 \\ t \neq=r}}^{l} \llbracket n_{s t} \mid x_{t}-a_{s t} \rrbracket\right) \llbracket n_{s r} \mid x_{r}-a_{s r} \rrbracket=w(\vec{x})
$$

is periodic $\bmod n_{0 r}$ as a function of $x_{r}$, by Theorem 1.3 we must have

$$
\sum_{\substack{s=1 \\ d_{r} \mid c_{r} n_{s r}}}^{k}\left(\lambda_{s} \prod_{\substack{t=1 \\ t \neq r}}^{l} \llbracket n_{s t} \mid x_{t}-a_{s t} \rrbracket\right) \frac{e^{2 \pi i\left(c_{r} / d_{r}\right) a_{s r}}}{n_{s r}}=0 .
$$

(Recall that $\left(c_{r} / d_{r}\right) n_{0 r} \notin \mathbb{Z}$.) 
Let $J=\left\{1 \leqslant s \leqslant k: d_{r} \mid c_{r} n_{s r}\right\}$ and $\lambda_{s}^{\prime}=\lambda_{s} n_{s r}^{-1} e^{2 \pi i a_{s r} c_{r} / d_{r}}$ for $s \in J$. Given $r^{\prime} \in\{1, \ldots, l\} \backslash\{r\}$ and $x_{t} \in \mathbb{Z}$ with $t \neq r, r^{\prime}$, we have

$$
\begin{gathered}
\sum_{s \in J}\left(\lambda_{s}^{\prime} \prod_{\substack{t=1 \\
t \neq r, r^{\prime}}}^{l} \llbracket n_{s t} \mid x_{t}-a_{s t} \rrbracket\right) \llbracket n_{s r^{\prime}} \mid x_{r^{\prime}}-a_{s r^{\prime}} \rrbracket \\
=\sum_{s \in J} \lambda_{s}^{\prime} \prod_{\substack{t=1 \\
t \neq r}}^{l} \llbracket n_{s t} \mid x_{t}-a_{s t} \rrbracket=0
\end{gathered}
$$

for all $x_{r^{\prime}} \in \mathbb{Z}$. By applying Remark $1.4 l-1$ times we finally obtain that

$$
\sum_{\substack{s=1 \\ \vec{d} \mid \vec{c} n_{s}}}^{k} \frac{\lambda_{s}}{n_{s 1} \cdots n_{s l}} e^{2 \pi i \sum_{t=1}^{l} a_{s t} c_{t} / d_{t}}=0 .
$$

Set $m=\min _{0 \leqslant s \leqslant k, \vec{d} \nmid \vec{n}_{s}}\left[d_{t} /\left(d_{t}, n_{s t}\right)\right]_{1 \leqslant t \leqslant l}$. Clearly $m \geqslant p\left(d_{1} \cdots d_{l}\right)$. Let $c$ be any positive integer less than $m$. For $s=0,1 \cdots, k$ we have

$$
\vec{d}\left|c \vec{n}_{s} \Leftrightarrow d_{t}\right| c n_{s t} \text { for all } t=1, \ldots, l \Leftrightarrow\left[\frac{d_{t}}{\left(d_{t}, n_{s t}\right)}\right]_{1 \leqslant t \leqslant l}|c \Leftrightarrow \vec{d}| \vec{n}_{s} .
$$

In other words, $\vec{d} \mid c \vec{n}_{s}$ if and only if $s \in I(\vec{d})$. (2.2) in the case $\vec{c}=\langle c, \ldots, c\rangle$ yields that

$$
\sum_{s \in I(\vec{d})} \frac{\lambda_{s}}{n_{s 1} \cdots n_{s l}} e^{2 \pi i c \sum_{t=1}^{l} a_{s t} / d_{t}}=0 .
$$

Let $\Theta=\left\{\left\{\sum_{t=1}^{l} a_{s t} / d_{t}\right\}: s \in I(\vec{d})\right\}$. Suppose that $|\Theta|<m$. Then for each $c=1, \ldots,|\Theta|$ we have

$$
\begin{aligned}
& \sum_{\theta \in \Theta} e^{2 \pi i c \theta} \sum_{\substack{s \in I(\vec{d}) \\
\left\{\sum_{t=1}^{l} a_{s t} / d_{t}\right\}=\theta}} \frac{\lambda_{s}}{n_{s 1} \cdots n_{s l}} \\
= & \sum_{s \in I(\vec{d})} \frac{\lambda_{s}}{n_{s 1} \cdots n_{s l}} e^{2 \pi i c \sum_{t=1}^{l} a_{s t} / d_{t}}=0 .
\end{aligned}
$$

By Lemma 2.1 this holds for all integers $c$, in particular $c=0$ :

$$
\sum_{s \in I(\vec{d})} \frac{\lambda_{s}}{n_{s 1} \cdots n_{s l}}=0 .
$$

This directly contradicts one of the hypotheses, whence $|\Theta| \geqslant m$. 


\section{Acknowledgment}

The author is indebted to the referee for his valuable suggestions to improve the presentation.

\section{References}

[CV1] R. B. Crittenden and C. L. Vanden Eynden, A proof of a conjecture of Erdös, Bull. Amer. Math. Soc. 75 (1969), 1326-1329.

[CV2] - Any $n$ arithmetic progressions covering the first $2^{n}$ integers cover all integers, Proc. Amer. Math. Soc. 24 (1970), 475-481.

[E1] P. Erdős, Remarks on number theory IV: Extremal problems in number theory I, Mat. Lapok 13 (1962), 228-255.

[E2] - Extremal problems in number theory, Proc. Sympos. Pure Math. 8 (1965), 181-189, Amer. Math. Soc., Providence, R. I..

[GJ] M. R. Garey and D. S. Johnson, Computers and Intractability: A Guide to the Theory of NP-completeness, W.H. Freeman, New York, 1983.

[N] M. Newman, Roots of unity and covering sets, Math. Ann. 191 (1971), 279-282.

[NZ] B. Novák, and Š. Znám, Disjoint covering systems, Amer. Math. Monthly 81 (1974), 42-45.

[P] Š. Porubský, Covering systems and generating functions, Acta Arith. 26 (1975), 223231.

[St] S. K. Stein, Unions of arithmetic sequences, Math. Ann. 134 (1958), 289-294.

[Su1] Z. W. Sun, Several results on systems of residue classes, Adv. Math. (China) 18 (1989), no. 2, 251-252.

[Su2] _ An improvement to the Znám-Newman result, Chinese Quart. J. Math. 6 (1991), no. 3, 90-96.

[Su3] On a generalization of a conjecture of Erdös, Nanjing Univ. J. Natur. Sci. 27 (1991), no. 1, 8-15.

[Su4] - Covering the integers by arithmetic sequences, Acta Arith. 72 (1995), 109-129.

[Su5] - Covering the integers by arithmetic sequences II, Trans. Amer. Math. Soc. 348 (1996), 4279-4320.

[Su6] Exact $m$-covers and the linear form $\sum_{s=1}^{k} x_{s} / n_{s}$, Acta Arith. 81 (1997), 175198.

[Su7] - Exact m-covers of groups by cosets, European J. Combin. 22 (2001), 415-429.

[Su8] , Algebraic approaches to periodic arithmetical maps, J. Algebra 240 (2001), $723-743$.

[Su9] On the function $w(x)=\left|\left\{1 \leqslant s \leqslant k: x \equiv a_{s}\left(\bmod n_{s}\right)\right\}\right|$, Combinatorica 23 (2003), 681-691.

[Su10] Unification of zero-sum problems, subset sums and covers of $\mathbb{Z}$, Electron. Res. Announc. Amer. Math. Soc. 9 (2003), 51-60.

[T] S. P. Tung, Complexity of sentences over number rings, SIAM J. Comp. 20 (1991), $126-143$.

Department of Mathematics (and Institute of Mathematical Science), Nanjing University, Nanjing 210093, The People's Republic of China

E-mail address: zwsun@nju.edu.cn Homepage: http://pweb.nju.edu.cn/zwsun 